\title{
Eine Mikromethode zur Proteinbestimmung im Serum nach der OUDIN-Technik
}

\author{
Von H. E. MülLER und I. MüLLER-von VorgT' \\ Aus der Medizinischen Klinik und Poliklinik der Berufsgenossenschaftlichen Krankenanstalten „Bergmannsheil“" Bocbum \\ (Direktor: Prof. Dr. E. Fritze)
}

(Eingegangen am 22. Dezember 1966)

Die Oudin-Geldiffusionstechnik wird als Mikromethode beschrieben und in ihrer Leistungsfähigkeit zur Proteinbestimmung in großen Reihen erprobt.

A micro-modification of the Oudin-gel diffusion technique is described. Its efficiency for the serial estimation of large numbers of proteins was tested.

Je weiter sich unsere Kenntnissë über die Pathophysiologie einzelner Krankheitsbilder vertiefen, um so interessanter wird die Bestimmung von Plasmaproteinen im Serum. Die Elektrophorese gestattet nur eine summarische Analyse der Eiweißkörper und einen groben Überblick. Detailliertere Methoden treten mehr und mehr in den Vordergrund und ermöglichen erst exakte Diagnosen krankhafter Zustände. Die Verfahren zur Bestimmung definierter Plasmaproteine lassen sich schematisch in folgende Gruppen fassen:

Die Bestimmung kann erfolgen unter Ausnutzung physiologischer Funktionen. Das ist etwa der Fall beim Coeruloplasmin, Haptoglobin und Transferrin (1), es ist möglich bei Präalbumin (2) oder Hämopexin (3).

Die Bestimmung kann erfolgen unter Ausnutzung unspezifischer, physikalisch-cbemischer Reaktionen oder Eigenschaften, wie beim Albumin (4), $\beta$-1-C-Globulin $(5,6)$ oder bei einigen anderen Glykoproteinen $(1,7)$, wo man sich der selektiven Denaturierung oder Resistenz gegen Denaturierung bedient, wie beim $\gamma$-GlobulinKomplex, dessen Löslichkeitseigenschaften $(1,7)$ in dest. Wasser, in Salzlösungen oder in wäßrigen Lösungen organischer Substanzen, wie Alkohol, quantitative Mengenangaben gestatten. Die Bestimmung kann erfolgen wie bei Lipoproteinen, die durch Polysulfate wie Heparin $(8,9)$, Dextransulfat $(5,6)$ oder ähnliche Substanzen präzipitiert oder in der Ultrazentrifuge $(1,7,10$, $11,12)$ abgetrennt und quantitativ erfaßt werden können. Auch spezielle Elektrophorese, Adsorption und Chromatographie an verschiedenen Trägern gehören hierher.

Die Bestimmung kann schließlich immunologisch erfolgen unter Ausnutzung der bei den einzelnen Plasmaproteinen verschiedenen, charakteristischen Antigenstrukturen. Allein die immunologische Bestimmung der Plasmaproteine gestattet eine schematisierende Methodik, während die physikalisch-chemische, quantitative Analyse für jedes einzelne Plasmaprotein eine spezielle Versuchsanordnung erfordert. Im Lauf der letzten Jahre wurden verschiedene immunologische Methoden zur Bestimmung der Plasmaproteine entwickelt. Sie basieren auf folgenden Prinzipien:

a) Die Antigen-Antikörper-Präzipitate werden unmittelbat während der Entstehung und Ausfällung nephelometriert (13-16).

1) Mit Unterstützung der Hohen Behörde der Montan-Union in Lux emburg. b) Das Präzipitatvolumen der ausgefallenen AntigenAntikörper-Komplexe wird als Immunokrit bestimmt (17).

c) Die methodische Anwendung der radioaktiven Verdünnungsanalyse gestattet die radioaktive Proteinbestimmung im Immunpräzipitat $(18,19,20)$.

d) Nach Heidelberger (21) wird der N-Gehalt des Präzipitats ermittelt. Das ist die älteste und auch heute noch exakteste Methode. Als Variation dieses Verfahrens kann die Proteinbestimmung etwa in der Biuret-Reaktion angesehen werden.

e) Bei den Agglutinationsmethoden in den Modifikationen der Hämagglutination, des Hämagglutinationshemmtests und entsprechender Latex-Reaktionen (21) läuft die Antigen-Antikörper-Reaktion an der Oberfläche von Partikeln ab. Diese Verfahren haben sich heute besonders bei Proteinen bewährt, die normalerweise nur in geringsten Konzentrationen oder nur unter pathologischen Bedingungen vorkommen, wie ProteinHormone, Rheumafaktoren, CRP u. ä.

f) Auf einem anderen Prinzip basieren die Immundiffusionsmethoden, die von Oudin, OUCHTERLONY und Elex eingeführt wurden (22). Hier bestimmt man den Diffusionsgradienten von Antigen und Antikörper in einem durchsichtigen Gel, meist in Agar. Grundsätzlich lassen sich zwei verschiedene Verfahren unterscheiden: Bei der einfachen Diffusionstechnik diffundiert nur einer der beiden Reaktionspartner, Antigen oder Antikörper, während der andere gleichmäßig im Gel verteilt ist. Bei der Doppeldiffusionstechnik diffundieren beide Komponenten in einem Gel. Bei jeder der beiden Techniken lassen sich wieder eine eindimensionale und eine zweidimensionale Methode unterscheiden. Oudin entwickelte die einfache, eindimensionale Immundiffusion im Röhrchen. Von FeINBerg (23) stammt die einfache zweidimensionale Immundiffusion auf einer Platte. Das OAKLEY-FulthORPE-Röhrchen (22) ist ein Beispiel für eine Doppeldiffusion der beiden Reaktionspartner in einer Dimension. Die OuchterlonY-Technik (22) schließlich ist eine zweidimensionale Doppeldiffusionsmethode, die in besonderem $\mathrm{Maß}$ Aussagen über kombinierte Systeme gestattet. Für die quantitative Proteinbestimmung empfehlen sich besonders die einfache einund zweidimensionale Geldiffusionstechnik in der Modifikation von Oudin oder FeInberg. 
In der vorliegenden Arbeit wurde die einfache, eindimensionale Agargeldiffusionsmethode nach OudrN als Mikromethode durchgeführt und für große Reihenuntersuchungen mit gleichzeitiger quantitativer Bestimmung von 12-16 Einzelproteinen modifiziert.

\section{Methodik}

Die zur Bestimmung der einzelnen Reinproteine verwendeten Antiseren wurden von den Behringwerken/Marburg bezogen: Anti-Präalbumin-Serum vom Kaninchen; Anti-Albumin-Serum vom Kaninchen; Anti- $\alpha_{1}$-Antitrypsin-Serum vom Kaninchen; Anti$\alpha_{1}$-Lipoprotein-Serum vom Kaninchen; Anti-saures- $\alpha_{1}$-Glykoprotein-Serum vom Kaninchen; Anti- $\alpha_{2}$-MakrogIobulin-Serum vom Kaninchen; Anti-Haptoglobin-Serum vom Kaninchen; AntiGc-Globulin-Serum vom Kaninchen; Anti- $\beta$-Lipoprotein-Serum vom Kaninchen; Anti- $\beta_{1}-C / \beta_{1}$-A-Globulin-Serum vom Kaninchen; Anti-Hämopexin-Serum vom Kaninchen; Anti-Transferrin-Serum vom Kaninchen; Anti- $\beta_{2}$-Glykoprotein-I-Serum vom Kaninchen; Anti- $\gamma$-A-Globulin-Serum von der Ziege; Anti- $\gamma$-M-GlobulinSerum vom Kaninchen; Anti- $\gamma$-G-Globulin-Serum vom Kaninchen.

Der Agar wurde 2-proz. in einem Diäthylbarbitursäure-AcetatPuffer, $\mathrm{pH} 8,2$ und Ionenstärke $\mu=0,07$ verwendet. Als Glasröhrchen wurden an beiden Enden offene Kapillaren benutzt, wie sie für die Mikrohämatokritbestimmung in Gebrauch sind: Länge $75 \mathrm{~mm}$, innerer Durchmesser $1,0 \mathrm{~mm}$.

Bei $50^{\circ}$ werden gleiche Volumenteile Agar und Antiserum vermischt. Diese Mischung steigt in den eingetauchten Kapillarröhtchen etwa $20-30 \mathrm{~mm}$ hoch. $2 \mathrm{~m} l$ Reaktionsmischung, jeweils $1 \mathrm{~m} l$ Agar $+1 \mathrm{~m} l$ Antiserum, gestatten so die Füllung von etwa 100 Kapillarröhrchen. Nach dem Erstarren bei Raumtemperatur stellt man die gefüllten Kapillarröhrchen, bei denen die Agarschicht unmittelbar mit dem Kapillarende abschließt, in ein Reagenzglas, das etwa $1 \mathrm{~m} l$ der zu untersuchenden Proteinlösung, Serum oder Serumverdünnung enthält. Während 48-stündigem Stehenlassen bei $4^{\circ}$ entstehen in den Röhrchen aufsteigende Präzipitatsäulen, deren Höhe im Agargel gemessen wird. Zur Ablesung dient eine Meßlupe der Fa. Bausch \& Lomb, Rochester, N.Y. mit linearer Skala und Stricheinteilung zu 0,1 mm. Entsprechend besteht eine Ablesungsgenauigkeit von $<0,1 \mathrm{~mm}$.

Zur Untersuchung gelangte ein Human-Sammelserum. Es wurde in Reagenzgläser gefüllt und bei $-20^{\circ}$ in einer Kühltruhe bis zu 4 Monaten gelagert. In dieser Zeitspanne erfolgte die Untersuchung des Serums, und zwar unverdünnt $(=1: 1)$ sowie $1: 2$, $1: 4,1: 8,1: 16$, und $1: 32$ verdünnt.

\section{Ergebnisse}

Folgende Faletoren wurden untersucht: die methodische Bedeutung des verdünnenden Agens, der Einfluß der Viskosität, der Agarkonzentration, der Höhe der Agarsäule und der Eintauchtiefe in die Probenmenge.

Die Verdünnung des Serums erfolgte mit physiologischer Kochsalzlösung und mit Normal-Kaninchen-Serum in geometrischen Verdünnungsreihen. Es zeigte sich, daß die Präzipitatzonen in Verdünnung mit Kaninchenserum stets geringer blieben als mit physiologischer $\mathrm{NaCl}$ Lösung. Daraus ergibt sich eine größere Genauigkeit bei Kochsalzverdünnung. Der mögliche Einfluß $\beta_{\text {der Vis- }}$ kosität auf die Höhe des Immunpräzipitats wurde durch Variation der Konzentration von zugesetztem Dextransulfat (Mol.-Gew. 500000) untersucht. Bis zu Werten von $\eta_{\mathrm{rel} .}=6,5$ (für Wasser $\eta_{\mathrm{rel} .}=1$ ) ergab sich keine Änderung der Präzipitathöhe.

Bei der Variation der Agar-Konzentration fanden sich in den Immundiffusionsröhrchen ansteigende Präzipitat- zonen mit sinkender Agarkonzentration. Niedrige Agarkonzentrationen erhöhen also die Diffusionshöhe und damit die Genauigkeit der Methode. Da die Probenmenge bzw. die Eintauchtiefe der Röhrchen wesentlichen Einfluß auf die Diffusionshöhe besitzt, wurde streng auf Volumenkonstanz der Untersuchungsproben geachtet. Dagegen hat die Höhe der Agarsäule im Immundiffusionsröhrchen keinen Einfluß auf die Diffusionshöhe. Wie aus Abbildung 1 und 2 hervorgeht, besteht eine logarithmische Abhängigkeit zwischen Immundiffusion und Antigen-Konzentration, also der Konzentration des zu untersuchenden Proteins. Ganz entscheidend wird die Höhe der Immundiffusionszone in den OudrN-Kapillaren durch die Qualität der Antiseren bestimmt. Auch die für die verschiedenen Proteine verschiedenen Streufehler sind durch die Qualität der Antiseren und die dadurch bedingte Ablesegenauigkeit der Höhe der Immundiffusionszone begrenzt. - Coeruloplasminbestimmungen konnten aus diesem Grund überhaupt nicht durchgeführt werden.

Da sich die Standardabweichung mit wachsender Verdünnung einem Endwert nähert, während der relative Fehler - Standardabweichung in \% der Mittelwerte mit wachsender Verdünnung ansteigt, ergibt sich für
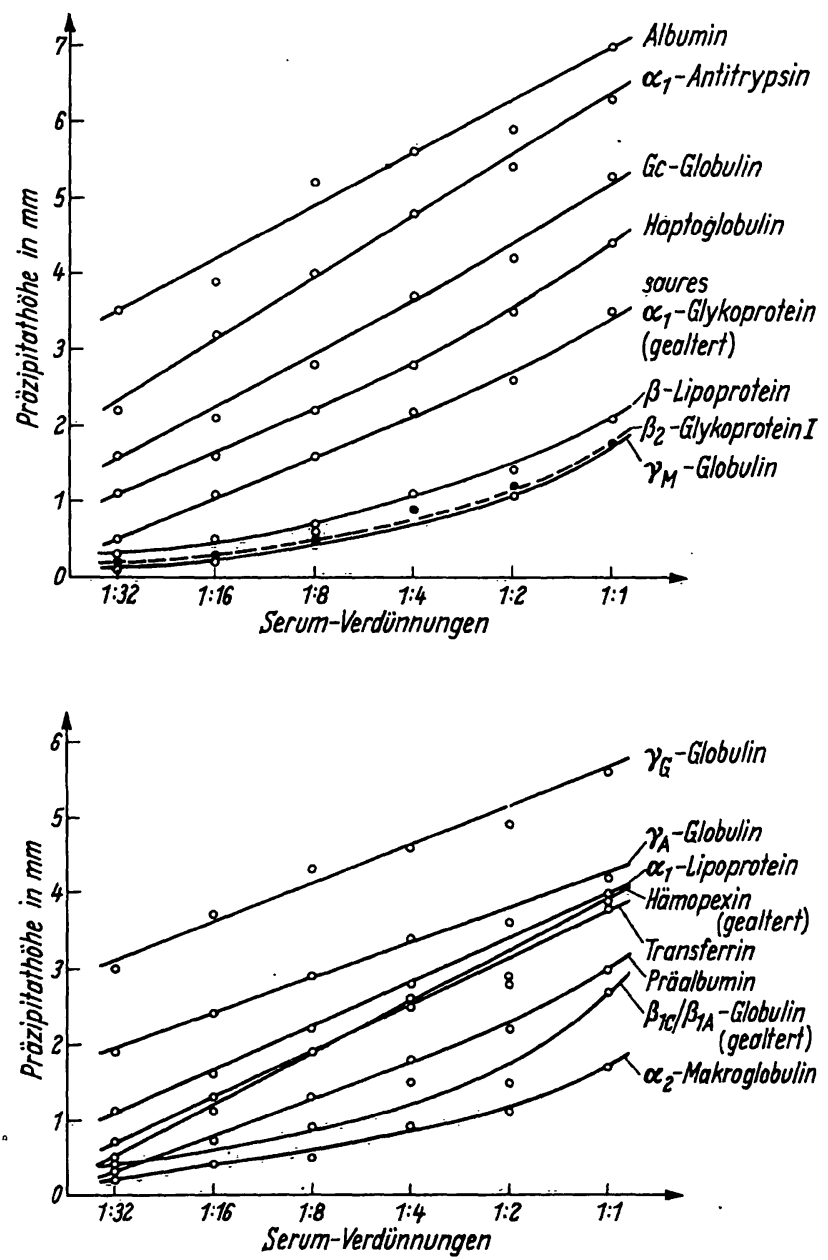

Abb. 1 und 2

Die Präzipitathöhen in den OudıN-Kapillaren in Abhängigkeit von den logarithmisch aufgetragenen Protein-Konzentrationen im Serum 
Tab. 1

Mittelwerte $(\bar{x})$ der Immundiffusionshöhen in den OudıN-Kapillaren (in mm) der einzelnen Serumproteine in Abhängigkeit von ihrer Konzentration (als Serum-Verdünnung) mit Standard-Abweichungen (s) und der Zahl der Messungen ( $\mathrm{n}$ )

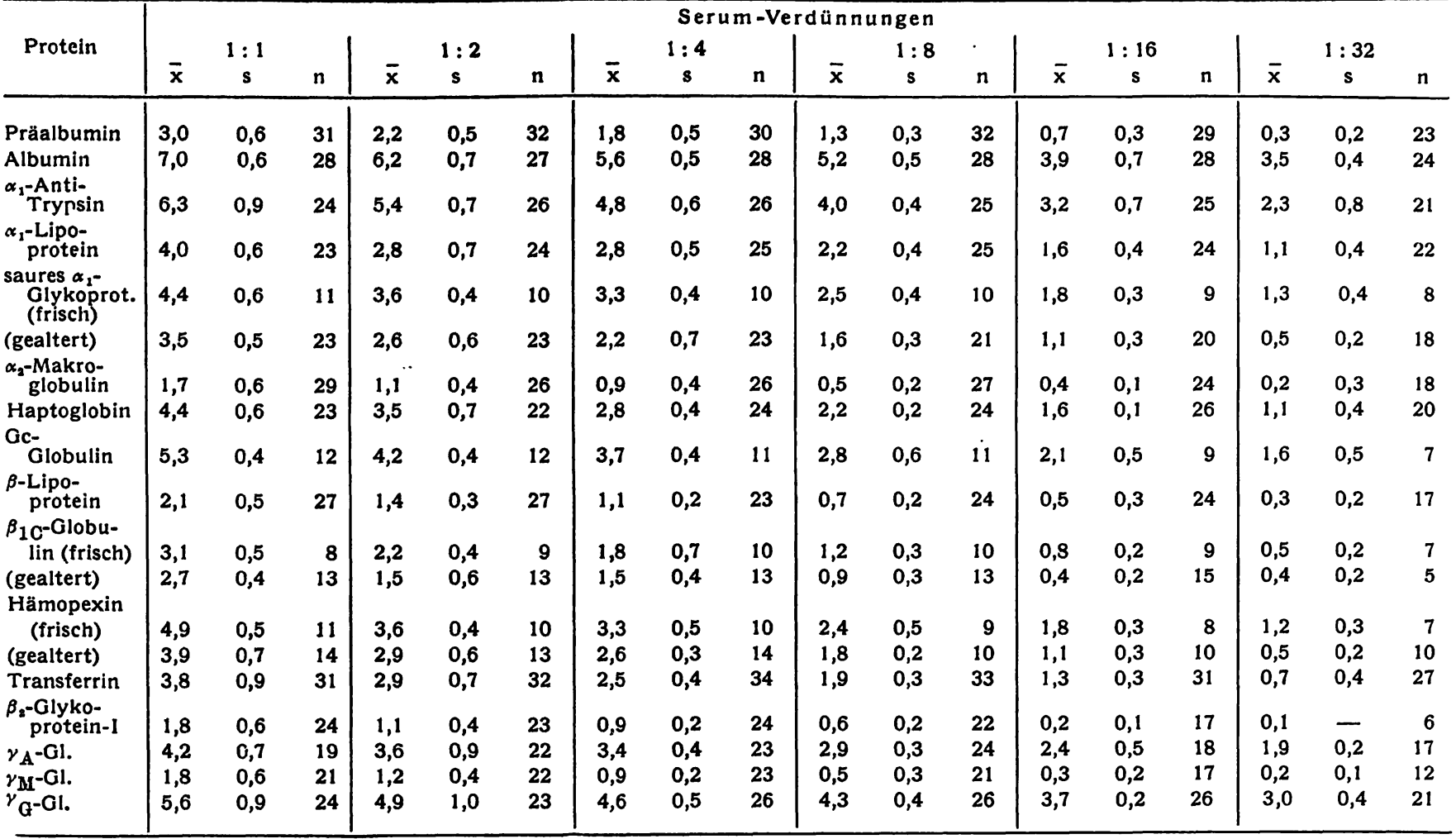

jede Proteinbestimmung eine optimale Konzentration. Um aber andererseits nicht mit zu vielen Serumverdünnungen arbeiten zu müssen, wurden von jeder Serumprobe zuei Gruppen gebildet und mit physiologischer Kochsalzlösung verdünnt, nämlich im Verbältnis 1:2 für die Bestimmung von: Präalbumin, saurem $\alpha_{1}$-Glykoprotein, $\alpha_{2}$-Makroglobulin, Gc-Globulin, $\beta$ Lipoprotein, Hämopexin, $\beta_{2}$-Glykoprotein-I, $\gamma$-MGlobulin. - Im Verbältnis 1:8 für die Bestimmung von: Albumin, $\alpha_{1}$-Antitrypsin, $\alpha_{1}$-Lipoprotein, Haptoglobin, $\beta_{1}-C / \beta_{1 A^{-}}$Globulin, Transferrin, $\gamma_{A^{-}}$Globulin, $\gamma_{G}$-Globulin.

Die Altersabhängigkeit des Serums wurde geprüft durch Gruppenbildung: Gruppe 1 wurde weniger als 20 Tage bei $-20^{\circ}$ gelagert und untersucht. Gruppe 2 wurde mehr als 35 Tage bei $-20^{\circ}$ gelagert und dann untersucht. Es ergaben sich mit der statistischen Sicherheitswahrscheinlichkeit von $99,5 \%$ aufgrund eines Vergleichs der beiden Mittelwerte Differenzen und damit Altersabhängigkeit beim sauren $\alpha_{1}$-Glykoprotein, beim $\beta_{1}-\mathrm{C} / \beta_{1 \mathrm{~A}}$-Globulin und beim Hämopexin. - Bei diesen drei Proteinen muß bei der Erstellung von Eichkurven (Abb. 1 und 2) auf diese Altersabhängigkeit geachtet werden.

\section{Diskussion}

Die angewandte Methodik begrenzt den Materialverbrauch und die Kosten auf ein Minimum. Es lassen sich mit $1 \mathrm{ml}$ Antiserum vermischt mit $1 \mathrm{ml}$ Agarlösung etwa 100 Mikrokapillaren füllen und damit bis zu 100 einzelne Proteinbestimmungen ausführen. In zwei Serumverdünnungsstufen (1:2 und 1:8) können praktisch alle bekannten Serumproteine bestimmt werden. Zweckmäßigerweise kennzeichnet man dazu die einzelnen Kapillaren mit unterschiedlichen wasserfesten Farbmarkierungen. Selbstverständlich wird die Methode erst dann interessant, wenn Reihenbestimmungen durchgeführt werden sollen. Als Einzeluntersuchung erfordert sie einen relativ großen Arbeitsaufwand, aber bei großen Kollektiven ist sie anderen Methoden überlegen. Das mag durch die Tatsache belegt werden, daß indessen in etwa 700 Serumproben etwa 10000 Einzelproteinbestimmungen durchgeführt wurden.

\section{Literatur}

1. Wuhrm and, F. und H. H. Märki, Dysproteinämien und Paraproteinämien, Grundlagen, Klinik und Therapie, Verlag Schwabe \& Co., Basel (1963). - 2. Woeber, K. A. und S. H. INGBAR, Endocrinology 75, 917 (1964). - 3. Schultze, H. E., K. Heide und H. HAUPT, Naturwissenschaften 48, 696 (1961). - 4. KaCHANI, Z. F. Ch., diese Z. 2, 91 (1964). - 5. LAURELI, C.-B. und B. LundH, Scand. J. Clin. Laborat. Invest. 14, 490 (1962). - 6. StrinBuCH; M., M. Quentin und L. PejAudier, Nature (London) 200, 262 (1963). - 7. Putnam, F. W., The plasma proteins, Academic Press New York-London (1960). - 8. Burstern, M. und J. Samartele, Clin. chim. Acta (Amsterdam) 3, 320 (1958). - 9. FrIED, R. und J. Hofflmayr, Klin. Wschr. 41, 246 (1963). - 10. Heide, K., R. SCHMIdtBerger und G. SchwrCK, Behringwerk-Mittlg., Heft 33, 96 (1957). - 11. Furman, R. H., S. S. Sanbar, P. Alaupóvic, R. H. BradFord und R. P. Howard, J. Laborat. Clin. Med., (S. Louis) 63, 193 (1964). - 12. LEVY, R. I. und D. S. FrEDRICKSON, J. Clin. Invest. 44, 426 (1965). - 13. Schultze, H. E und G. SCHwiCK, Behringwerk-Mittlg. Heft 35, 57 (1958). -14. .SCHULtzE H. E. und G. Schwick, Clin. chim. Acta (Amsterdam), 4, 15 (1959). - 15. HöLZER, K. H. und G. Binzus, 69. Verh. Dtsch. Ges. 
inn. Med. S. 456 (1963). - 16. Hawkrns, J. D., Immunology 7, 229 (1964). - 17. Alexander H. L., und M. B. Johnson und J. H. Alexander, Science (Wash.) 101, 547 (1945.) - 18. Weiler, R. J., D. Hofstra, A. Szenivanyi, Rt. Blaisdell und D. W. Talmage, J. Inmunology 85, 130 (1960). - 19. FAHEY, J. L. und M. E. LaWrence, J. Immunology 91, 597 (1963). - 20. MCKeLVEy, E.
M. und J. L. FAHEY, J. Clin. Invest. 44, 1778 (1965). - 21. KABAT, E. A. und M. M. MAYER, Experimental Immunochemistry, 2. Aufl. Ch. C. Thomas, Springfield, Ill. (1961). - 22. Ouchrerlony, Ö., Immunchemie, 15. Colloqu. der Ges. f. physiol. Chemie, Mosbach/ Baden 1964, Springer-Verlag, Berlin-Heidelberg-New York (1965). 23. Fennberg, J. G., Internat.Arch. Allergy 11, 129 (1957).

Dr. Dr. H. E. Müller 34 Göttingen, Kreuzbergring 57

\title{
Fermente des menschlichen Blutes
}

\section{Mitteilung1): Quantitative Verfolgung des Wirkungsverlaufes von Bernsteinsäuredicholinester und Bernsteinsäuremono- cbolinester auf esterspaltende Enz'J'me des menschlicben Blutes}

\author{
Von W. Pilz, A. T. Boo und E. StelzL \\ Aus dem Pbysiologisch-chemischen und analytischen Labor (Leiter: Dr. W. Pilz) der Ärqtlichen Abteilung \\ (Leiter: Dr. H. Hörlein) der Farbenfabriken Bayer AG, Werk Leverkusen
}

(Eingegangen am 3. September 1966)

\begin{abstract}
Es wurde der Verlauf der Hemmwirkung von Bernsteinsäuredicholinester und Bernsteinsäuremonocholinester auf die Serumesterasen Acetylcholinesterase, Carboxylesterase, die Arylesterasen, die endogene Lipoproteidlipase sowie auf die Acetylcholinesterase menschlicher Erythrocyten quantitativ verfolgt. Es ergaben sich zum Teil stark unterschiedliche Hemmkurven. Die Acetylcholinesterasen in Serum und Erythrocyten zeigen ein ungewohntes Bild. Aus der Identität der Kurvenverläufe konnte dịe große Ähnlichkeit von 3 Acetylcholinesterasetypen erkannt werden. Der stark unterschiedliche Kurvenverlauf für beide Arylesterasen erhärtet den schon früher auf anderem Weg erhobenen Befund, daß es sich dabei um verschiedene Enzyme handelt.
\end{abstract}

The time course of inhibition by succinic acid dicholine ester and succinic acid monocholine ester was measured quantitatively on the following serum enzymes: acetylcholine esterase, carboxylesterase, arylesterase, endogenous lipoprotein lipase, and on the acetylcholine esterase of human erythrocytes. There were some marked differences in the inhibition-curves. The acetylcholine esterases in serum and erythrocytes give an unusual picture. The identical nature of the curves indicates a strong similarity between the 3 acetylcholi.e esterase types. The markedly different curves for the two arylesterases strengthens the earlier finding, obtained by different means, that these are separate enzymes.

Wie wir zeigen konnten, wird Bernsteinsäuredicholinester von menschlichem Vollserum nicht hydrolysiert (2). Diese Befunde stehen im Widerspruch zu der Theorie von KaLow, der im menschlichen Vollserum eine deutliche Hydrolysefähigkeit für Bernsteinsäuredicholinester fand. Die bei der Applikation der Verbindung gelegentlich auftretenden verlängerten Apnoen werden auf eine atypische Pseudocholinesterase zurückgeführt, die nach KALOw (3) genetisch determiniert ist (Literatur s. (3)). Eine Reihe von Fakten sprechen gegen diese Theorie. So haben von den insgesamt 104 von Kalow beobachteten Apnoen 35 Fälle einen normalen Enzymspiegel und 4 Fälle eine zwar erniedrigte Enzymaktivität, aber normale Dibucainzahlen; somit konnten 39 Fälle als dem normalen Gentyp zugehörig identifiziert werden (3) (vgl. A. Doenicke (4)). Nach diesen Resultaten können verlängerte Apnoen nach Succinylbischolin-Applikation nicht mehr ausschließlich auf das Vorhandensein einer genetisch determinierten atypischen Pseudocholinesterase oder einer Mischform zwischen dieser und einem normalen Enzym (intermediäre Esterase) zurückgeführt werden. Die Identifizierung einer atypischen Pseudocholinesterase (Substrat: Benzoylcholin!)2) erfolgt $z$. B.

1) I.ctzte Mitteilung: W. Pilz, Hoppe Seyler's Z. physiol. Chem. 345, 80 (1966).

2) Enzym: E. C. 3.1.1.9 - Dieses Enzym ist nach neuesten Erkenntnissen (1) mit dem Enzym E. C. 3.1.1.8. identisch; es handelt sich um eine Enzymgruppe (11 Einzelfermente), die substratunspezifisch ist. durch die Bestimmung der Dibucainzahl. Auch Mischformen können so erfaßt werden (3). Wir werden in Kürze beweisen, daß die Bestimmung der Dibucainzahlen $(5,6,7)$ zumindest von analytischer Seite her problematisch ist (8).

Nach den Gesetzen der Genetik ist beim heutigen Stand unserer Kenntnisse die Annahme eines stummen Gens (,silent gene") notwendig. Im Arbeitskreis von GoEDDE் (7) konnte vor kurzem nachgewiesen werden, daß ein Serum, das als Vollserum überhaupt keine enzymatische Aktivität für Acetylcholin und Benzoylcholin aufwies, nach Trennung im Stärkegel deutliche enzymatische Aktivitäten hatte, die von denen eines Normalserums kaum verschieden waren. Somit dürfte die Annahme eines stummen Gens als nicht gesichert gelten (vgl. dazu die Hypothese eines die Serumesterasen hemmenden Proteins, das eine andere elektrophoretische Wanderungsgeschwindigkeit als die Enżyme hat (2)).

Die extrem kurze Wirkungsdauer von Succinylbischolin in vivo kann mit einer Hydrolyse des Enzyms durch menschliche Serumesterasen nicht erklärt werden. Dagegen konnten wir vor kurzem an 68 verschiedenen Lungenhomogenaten zeigen, $\mathrm{da} ß$ in der menschlichen Lunge das Gleichgewicht der Reaktion Succinylbischolin $+\mathrm{H}_{2} \mathrm{O} \rightleftharpoons$ Succinylmonocholin + Cholin ganz auf der Seite des Succinylmonocholins liegt (10). Die Einstellung des Gleichgewichtes erfolgt unter Verhältnissen, die den Bedingungen in vivo nachgeahmt sind, durch ein noch nicht isoliertes Enzym innerhalb 